\title{
The Hybrid Approach of LCO and SA to Solve Job-shop Scheduling Problem*
}

\author{
Yasumasa TAmura ${ }^{\dagger}$, Ikuo SuzukI ${ }^{\ddagger}$, Masahito YAmamoto $^{\dagger}$ and Masashi FurukawA ${ }^{\dagger}$
}

\begin{abstract}
A Job-shop Scheduling Problem (JSP) is one of the combinatorial optimization problems. JSP appears as a basic scheduling problem in many situations of a manufacturing system and many methods for JSP have been invented. This study examines two effective methods, SA and LCO, for JSP and propose a hybrid method based on them. As a result of the experiments, the proposed method can find a good solution with short computational time. Summarizing this study, the proposed method is efficient in the early or middle search of the optimization.
\end{abstract}

\section{Introduction}

A Job-shop Scheduling Problem (JSP) is to determine schedules for operating several jobs on several machines. An objective of JSP is to find a good assignment of jobs to machines. Generally, such an assignment means the efficient schedule by which all jobs are operated in the shortest time. JSP appears in many occasion of manufacturing. For instance, JSP can be applied not only to a simple manufacturing schedule problem but also to a complex manufacturing schedule problem with additional constraints.

Many methods have been invented to solve JSP, and some of them are called "exact method". For example, the branch and bound method is one of the most famous exact methods. The exact method means it can search for the optimal solution, when all jobs are operated in the shortest time. However, most of all exact methods request large memory and time-consuming because JSP is not only categorized in NP-hard class shown by Garey and Jhonson[1] but also known as one of the most difficult NP-hard class members. Such properties are not reasonable because there is a due time in a real industrial world. Thus, though the exact solution methods can derive the ideal schedule, they are not reasonable to solve JSP in the real industrial world.

Instead of the exact methods, there have been many investigations to solve JSP with reasonable time. Heuristic approach is one of those investigations. Though the schedule generated by the heuristic is not

\footnotetext{
* Manuscript Recieved Date : May 25, 2012

$\dagger$ Graduate School of Information Science and Technology, Hokkaido University; Sapporo, Hokkaido 060-0814, JAPAN

¥ Department of Computer Science, Kitami Institute of Technology; Kitami, Hokkaido 090-8507, JAPAN

Key Words: combinatorial optimization problem, job-shop scheduling problem, meta-heuristics, Simulated Annealing, Local Clustering Organization, hybrid solution.
}

optimal, it is known that the heuristic can derive the schedule highly close to optimal one. Genetic Algorithm (GA) is one of the most famous heuristics in the field of optimization. Applying GA to JSP is investigated actively and most investigations adopting GA or extended GA indicate their effectivity. Simulated Annealing (SA) is proved to be available for solving JSP [3]. In fact, because it is widely known that standard SA efficiently derives much better schedule than the standard GA, SA is the effective method except its difficulty to use. Local Clustering Organization (LCO) is a new heuristic invented for a large scale Traveling Salesman Problem (TSP)[4]. And some studies prove its availability for large scale JSP[5][6]. LCO is a kind of local search methods, which is superior to GA in terms of the convergence. In addition, LCO uses some clustering methods to improve a provisional solution, and each method has different property for searching a better one. For these reasons, to solve the sequencing problems such as TSP and JSP, it is proved LCO is more effective than GA.

In LCO, the optimal solution is searched by using a simple improvement mechanism called "hill climbing improvement". However, such an improvement mechanism causes a risk which the solution falls into the local optima. It is a weakness of LCO. To avoid the risk, SA has a mechanism to accept worse solutions stochastically. Therefore, to improve LCO, we propose a hybrid which is composed by SA and LCO in this paper.

In order to propose the hybrid method, the rest of the paper is organized as follows. Section $\mathbf{2}$ describes important features of JSP. Two algorithms used in this study are explained in Section 3. Section 4 proposes a solution on JSP. Results are compared between the new solution and other ones in Section 5. Section 6 summarizes this study. 


\section{Problem and Solution Model}

JSP is a combinatorial optimization problem to obtain an efficient schedule for processing some jobs by some machines. Therefore, a set of jobs and a set of machines are given as a problem instance.

The following notations are used for representing JSP. Let $n$ and $m$ be the number of jobs and the number of machines.

$$
\begin{array}{cl}
J=\left\{J_{i}\right\} & : \quad \text { a set of jobs }(1 \leq i \leq n) \\
M=\left\{M_{k}\right\} & : \quad \text { a set of machines }(1 \leq k \leq m)
\end{array}
$$

Some constraints are given as follows;

(1) Each job has some operations processed by specified machines. And the sequence of operations is independently given to each jobs.

(2) Every operation is not interrupted and resumed in processing.

(3) When a job is processed by a machine, the job is not processed by any other machines simultaneously.

(4) When a machine processes a job, any other jobs are not set to the same machine simultaneously.

From the constraint $1, J_{i}$ is defined as eq. (1). Here, $j_{i}^{x}$ indicates the $x^{\text {th }}$ operation of $J_{i}$.

$$
J_{i}=\left(j_{i}^{1}, j_{i}^{2}, \cdots, j_{i}^{m}\right)
$$

Also, we define some mappings used for presenting a mathematical model of JSP.

$$
\begin{array}{lll}
S\left(j_{i}^{x}\right) & : & \text { start time of } j_{i}^{x} \text { (decision variable) } \\
P\left(j_{i}^{x}\right) & : \text { processing time of } j_{i}^{x} \\
R\left(j_{i}^{x}\right) & : \quad \text { machine in which } j_{i}^{x} \text { is processed }
\end{array}
$$

In general, $P(*)$ and $R(*)$ is given by each instance of JSP, and the efficiency of a schedule is evaluated by makespan. From the constraint 2, the termination time of $j_{i}^{x}$ is determined as

$$
S\left(j_{i}^{x}\right)+P\left(j_{i}^{x}\right)
$$

Therefore, the makespan is defined as eq. (3).

$$
\text { makespan }=\max _{i}\left\{S\left(j_{i}^{m}\right)+P\left(j_{i}^{m}\right)\right\}
$$

From these notations, constraints and equations, the mathematical model of JSP by using the equations from eq. (4) to eq. (6). Here, eq. (4) represents the objective of JSP, eq. (5) and eq. (6) represent the constraint 3 and 4 .

$$
\begin{aligned}
& \operatorname{minimize} \max _{i}\left\{S\left(j_{i}^{m}\right)+P\left(j_{i}^{m}\right)\right\} \\
& \text { subject to } \\
& S\left(j_{i}^{x+1}\right) \geq S\left(j_{i}^{x}\right)+P\left(j_{i}^{x}\right) \\
& (1 \leq i \leq n, 1 \leq x<m) \\
& S\left(j_{i}^{x}\right) \geq S\left(j_{k}^{y}\right)+P\left(j_{k}^{y}\right) \\
& \left(1 \leq i, k \leq n, 1 \leq x, y \leq m, R\left(j_{i}^{x}\right)=R\left(j_{k}^{y}\right)\right)
\end{aligned}
$$

\subsection{Instance}

Generally, a simple instance of JSP is represented as Table 1 . In this case, the instance is called the $3 \times 3$
Table 1 An instance of JSP

\begin{tabular}{|c|c|c|c|}
\hline- & 1 & 2 & 3 \\
\hline$J_{1}$ & $\left(M_{1}, 12\right)$ & $\left(M_{2}, 23\right)$ & $\left(M_{3}, 9\right)$ \\
\hline$J_{2}$ & $\left(M_{2}, 25\right)$ & $\left(M_{1}, 10\right)$ & $\left(M_{3}, 5\right)$ \\
\hline$J_{3}$ & $\left(M_{2}, 10\right)$ & $\left(M_{3}, 16\right)$ & $\left(M_{1}, 22\right)$ \\
\hline
\end{tabular}

\begin{tabular}{|l|l|l|l|l|l|l|l|l|}
\hline$J_{3}$ & $J_{1}$ & $J_{2}$ & $J_{3}$ & $J_{1}$ & $J_{2}$ & $J_{2}$ & $J_{1}$ & $J_{3}$ \\
\hline
\end{tabular}

(a) Solution representation

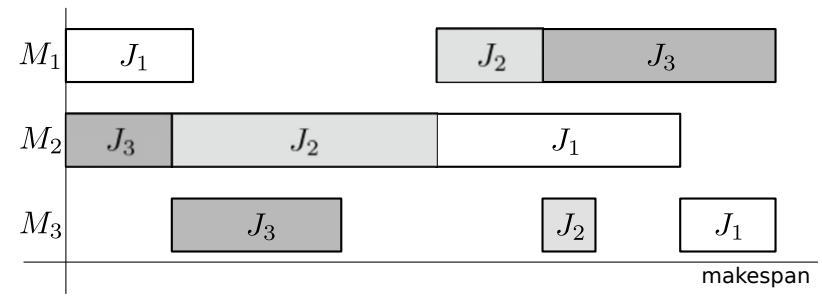

(b) Gantt chart

Fig. 1 A solution for the instance

problem because it has three jobs and three machines. Similarly, if a problem has $n$ jobs and $m$ machines, then the problem is called the $n \times m$ problem. In Table 1, for example, $\left(J_{1}, 1\right)=\left(M_{1}, 12\right)$ means that $\left(J_{1}, 1\right)$ is processed by $M_{1}$ and its processing time is 12. Also, that means $R\left(j_{1}^{1}\right)=M_{1}$ and $P\left(j_{1}^{1}\right)=12$.

\subsection{Solution Representation}

Fig. 1(a) represents one of solutions we adopted. It corresponds to the problem given by Table 1 . The instance has 3 jobs and each job has 3 operations on 3 machines. Each entity in the solution represents each job and the same entity appears the same times as the number of machines.

The solution representation is transformed into Gantt Chart, using the follow procedure. Here, $d$ means an id of a solution entity, $\lambda$ means a job number and $\theta_{\lambda}$ means an id of the operation in $J_{\lambda}$.

(1) Set $\theta_{i}=1(\forall i \in \mathbb{N}, 1 \leq i \leq n)$ and $d=1$

(2) Get the $d^{\text {th }}$ entity, which is $J_{\lambda}(1 \leq \lambda \leq n)$, from the solution representation.

(3) Calculate $S\left(j_{\lambda}^{\theta_{\lambda}}\right)$ and allocate $j_{\lambda}^{\theta_{\lambda}}$ to Gantt Chart

(4) $\theta_{\lambda} \leftarrow \theta_{\lambda}+1, d \leftarrow d+1$

(5) If $d \leq n \times m$, which means the length of the solution representation, go back to Step 2.

Adapting this procedure, the solution representation shown in Fig. 1(a) is transformed into the schedule representation shown in Fig. 1(b).

\section{Fundamental Algorithms for a $\mathbf{H y -}$ brid Method}

This paper proposes a hybrid method on JSP. The method is the hybrid algorithm consisting of two meta-heuristic algorithms, SA and LCO. We describe two algorithms before proposing the hybrid method. 


\subsection{Simulated Annealing (SA)}

$\mathrm{SA}$ is a probabilistic meta-heuristic, and a neighborhood search algorithm. It is highly important for SA that it tries to explore a global optima by not only improving a solution, but also stochastically taking worse one.

Notations to explain SA is as follows;

$\begin{array}{cl}T(t) & : \text { the temperature at } t^{\text {th }} \text { step } \\ I(t) & : \text { the number of iterations at } t^{\text {th }} \text { step } \\ S_{b} & : \text { the best solution } \\ S_{c} & : \text { the current solution } \\ S_{n} & : \text { the neighborhood solution } \\ C(S) & : \text { the cost of a solution } S\end{array}$

As an algorithm to minimize $C(S)$, SA's procedure is described under-below.

(1) Set $t=0$.

(2) Initialize $S_{c}$ at random.

(3) Set $S_{b}=S_{c}$.

(4) Generate $S_{n}$

(5) Set $\Delta_{n c}=C\left(S_{n}\right)-C\left(S_{c}\right)$ and $\Delta_{n b}=C\left(S_{n}\right)-$ $C\left(S_{b}\right)$.

(6) If $\Delta_{n b}<0$, then replace $S_{b}$ and $S_{c}$ with $S_{n}$.

(7) If $\Delta_{n c} \leq 0$, then replace $S_{c}$ with $S_{n}$. Otherwise generate an uniform random value $P(\forall P \in$ $\mathbb{R}, 0 \leq P \leq 1)$ and replace $S_{c}$ with $S_{n}$ if $P^{\prime}<P$. Here, $P^{\prime}$ is defined as;

$$
P^{\prime}=\exp \left\{-\frac{\Delta_{n c}}{T(t)}\right\}
$$

(8) Iterate step 4-7 $I(t)$ times.

(9) Replace $t$ with $t+1$.

(10) If terminate conditions are satisfied, terminate this procedure with the solution $S_{b}$. Otherwise go back to step 4.

In the procedure, we do not describe the neighborhood solution, the relation between time steps and the temperature, and the maximum iteration number. We discuss the neighborhood solution and parameters. In addition to that, we consider the advantage and the disadvantage of SA.

\subsubsection{Neighborhood Solution}

In the procedure, at step 3, a neighborhood solution $S_{n}$ is generated by using an operation selected stochastically from three operations. They are defined as follows.

\section{- Swapping}

(1) Select two elements in $S_{c}$ at random.

(2) $S_{n}$ is generated by swapping those selected elements in $S_{c}$.

\section{- Inverting}

(1) Select two elements in $S_{c}$ at random.

(2) $S_{n}$ is generated by inverting the alignment of all elements which is allocated between two selected elements in $S_{c}$.

\section{- Inserting}

(1) Select two elements in $S_{c}$ at random.

(2) $S_{n}$ is generated by removing the backward element from $S_{c}$ and inserting it in front of another element in $S_{c}$.

\subsubsection{Temperature Parameter}

In SA, the cooling schedule, which means how to decrease the temperature per step, is one of the most important parameters for effectively solving the problem. From some researches about SA, it is reported that "logarithmic cooling", shown in eq. (8), is usually effective.

$$
T(t)=\frac{T(0)}{\log _{2}(1+t)}
$$

However, the logarithmic cooling requires long computation time for derive a solution. Therefore, the logarithmic cooling is rarely used. And many studies use "geometric cooling" shown in eq. (9) with use of the decreasing rate $d T$.

$$
T(t+1)=T(t) \times d T
$$

In this study, we use the geometric cooling as the cooling schedule.

\subsubsection{Iteration Parameter}

If $I(t)$ is a suitable value, it can search for the neighborhoods adequately. However, finding such $I(t)$ is very difficult because it effects on other related parameters and factors such as the size of the problem or the length of the processing time each process has. Therefore, simply defined $I(t)$ is generally used. The following shows our definition.

$$
I(t+1)= \begin{cases}I(t) \times d I & I(t+1)<I_{\max } \\ I_{\max } & \text { otherwise }\end{cases}
$$

On eq. (10), $I_{\max }$ is the upper limit of $I(t)$ and $d I$ is the increasing rate of $I(t)$.

\subsubsection{Some Remarks on SA}

In many cases, we can obtain a solution nearly closer to the optima by using SA. However there exist some problems as follows.

- It takes a long time to acquire a better solution in a large scale problem.

- In any cases, it is difficult to adjust some parameters.

\subsection{Local Clustering Organization (LCO)}

LCO is a meta-heuristic algorithm proposed by Furukawa et al.[4]. For large-scale TSP, it is reported LCO gives a highly accurate approximate solution with fast computation time. In LCO, optimization is performed based on the Riccati-type learning equation. The Riccati-type learning equation is used for Self-Organizing Map (SOM) which is proposed by Kohonen[7]. SOM is capable of deriving a highly accurate approximate solution with fast computational time on TSP, but it needs cities coordinates as synapses vectors. On the other hand, LCO does not need them. Therefore LCO is the more general algorithm on TSP. 
Because LCO is a meta-heuristic algorithm, we can use it for solving not only TSP but also the other combinatorial optimization problems. On JSP, as suggested in the introduction, it is proved LCO is capable of deriving a highly accurate approximate solution with fast computational time by comparing it with Genetic Algorithm (GA).

Here, we introduce some notations to explain LCO.

$\begin{array}{cl}r(t) & : \text { the clustering radius in } t^{\text {th }} \text { step } \\ S_{c} & : \text { the current solution } \\ S_{n} & : \text { the neighborhood solution } \\ C(S) & : \text { the cost of a solution } S\end{array}$

The optimization (minimization) procedure of LCO is described as follows;

(1) Set $t=0$.

(2) Initialize $S_{c}$ at random.

(3) Randomly select a set of consecutive entities in $S_{c}$ whose size is equal to $2 r(t)+1$.

(4) To generate $S_{n}$, apply a clustering operation to the selected set.

(5) If $C\left(S_{n}\right)$ is better than $C\left(S_{c}\right)$, replace $S_{c}$ with $S_{n}$ generated by the clustering operation.

(6) Replace $t$ with $t+1$.

(7) If terminate conditions are satisfied, stop the procedure. Otherwise, go back to step 3.

According to the procedure above, generating $S_{n}$ mostly depends on the clustering operation. Also, the clustering radius has a great effect on the result of optimization. There are no explanations about the operation or the clustering radius. In addition, there is a room to improve LCO. In the following sections, we describe the clustering operation and radius in detail.

\subsubsection{Clustering Operation}

The clustering method is one of the most important parts in LCO and optimization is performed by applying it to a part of solution selected randomly. In this paper, we use four methods to avoid falling into a local optimal solution from which it is hard to escape. These methods are oriented to beneficial operations on JSP. The additional notations are introduced to explain clustering methods under below.

- $d_{c}$ : the id of the center entity in selected local

- $S_{c}[d]$ : the $d^{\text {th }}$ entity in $S_{c}$

And here, $i$ is used for counter variables, not for job ID's.

\section{- Simple Exchange Method (SEM)}

Fig. 2(a) indicates a behavior of SEM. In this method, $S_{n}$ is generated by swapping two entities in $S_{c}$. The algorithm is described as follows;

(1) Set $i=1$.

(2) Create $S_{n}$ by swapping $S_{c}\left[d_{c}\right]$ for $S_{c}\left[d_{c}-i\right]$.

(3) If $C\left(S_{n}\right)<C\left(S_{c}\right)$, then replace $S_{c}$ with $S_{n}$.

(4) Create $S_{n}$ by swapping $S_{c}\left[d_{c}\right]$ for $S_{c}\left[d_{c}+i\right]$.

(5) If $C\left(S_{n}\right)<C\left(S_{c}\right)$, then replace $S_{c}$ with $S_{n}$.

(6) Replace $i$ with $i+1$.

(7) If $i \leq r(t)$, then go back to the step 2. Otherwise stop the procedure.

\section{- Symmetrical Exchange Method (SYM)}

Fig. 2(b) indicates a behavior of SYM. In this method, $S_{n}$ is generated by swapping two elements in $S_{c}$ as well as SEM. However, two entities are located at the symmetrical position on the entity which occupies at the center of the local solution. The algorithm is explained as follows;

(1) Set $i=1$.

(2) Create $S_{n}$ by swapping $S_{c}\left[d_{c}-i\right]$ for $S_{c}\left[d_{c}+\right.$ $i]$.

(3) If $C\left(S_{n}\right)<C\left(S_{c}\right)$, replace $S_{c}$ with $S_{n}$.

(4) Replace $i$ with $i+1$.

(5) If $i \leq r(t)$, go back to the step 2. Otherwise stop the procedure.

\section{- Inverse Exchange Method (IEM)}

Fig. 2(c) indicates a behavior of IEM. In this method, $S_{n}$ is generated by inverting the arraignment between two entities in $S_{c}$. The algorithm is explained as follows;

(1) Set $i=1$.

(2) Create a $S_{n}$ by inverting the arraignment between $S_{c}\left[d_{c}\right]$ and $S_{c}\left[d_{c}-i\right]$.

(3) If $C\left(S_{n}\right)<C\left(S_{c}\right)$, then replace $S_{c}$ with $S_{n}$.

(4) Create $S_{n}$ by inverting the arraignment between $S_{c}\left[d_{c}\right]$ and $S_{c}\left[d_{c}+i\right]$.

(5) If $C\left(S_{n}\right)<C\left(S_{c}\right)$, replace $S_{c}$ with $S_{n}$.

(6) Replace $i$ with $i+1$.

(7) If $i \leq r(t)$, go back to the step 2. Otherwise stop the procedure.

\section{- Simple Shift Method (SSM)}

In this method, the shift change is given to $S_{c}$. Fig. 2(d) describes a behavior of SSM. The algorithm is explained as follows;

(1) Set $i=1$.

(2) Create $S_{n}$ by moving $S_{c}\left[d_{c}-r(t)+i\right]$ to the left of $S_{c}\left[d_{c}-r(t)\right]$.

(3) If $C\left(S_{n}\right)<C\left(S_{c}\right)$, then replace $S_{c}$ with $S_{n}$.

(4) Replace $i$ with $i+1$.

(5) If $i \leq d_{c}+r(t)$, then go back to the step 2 . Otherwise terminate procedure.

\section{- Smoothing Method (SM)}

Fig. 2(e) describes a behavior of SM. This method performs an operation on all combinations of two entities within the local selected entity sequence, and the exchanging method or the shifting method is generally used as the operation. In this study, we use the exchanging method. The algorithm is defined as follows;

(1) Set $i=0$.

(2) Set $j=i+1$

(3) Create $S_{n}$ by exchanging $S_{c}\left[d_{c}-r(t)+i\right]$ with $S_{c}\left[d_{c}-r(t)+j\right]$.

(4) If $C\left(S_{n}\right)<C\left(S_{c}\right)$, replace $S_{c}$ with $S_{n}$.

(5) Replace $j$ with $j+1$.

(6) If $j \leq d_{c}+r(t)$, go back to the step 3. Otherwise go to next step.

(7) Replace $i$ with $i+1$. 


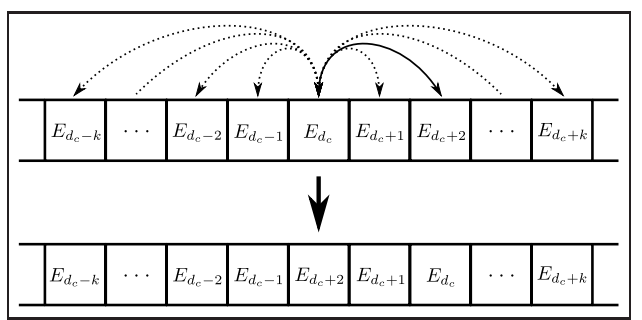

(a) $\operatorname{SEM}(k=r(t))$

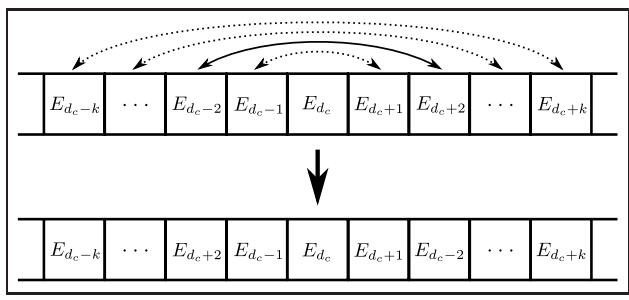

(b) SYM $(k=r(t))$

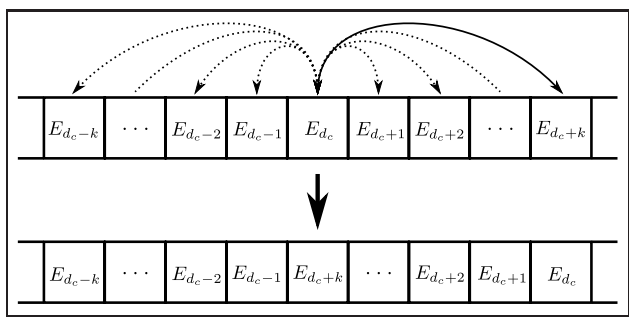

(c) $\operatorname{IEM}(k=r(t))$

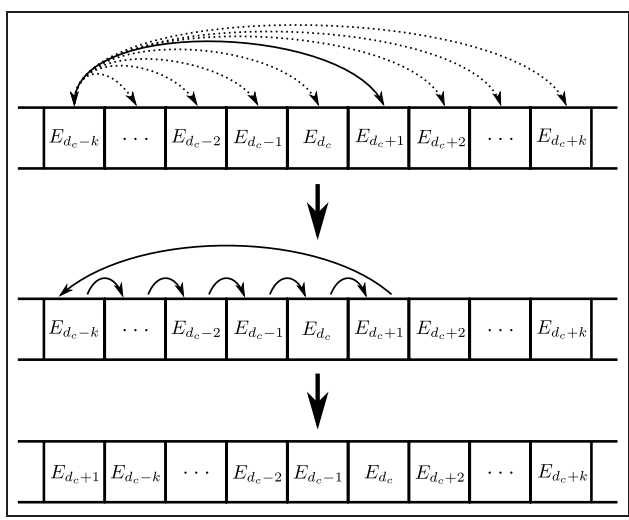

(d) $\operatorname{SSM}(k=r(t))$

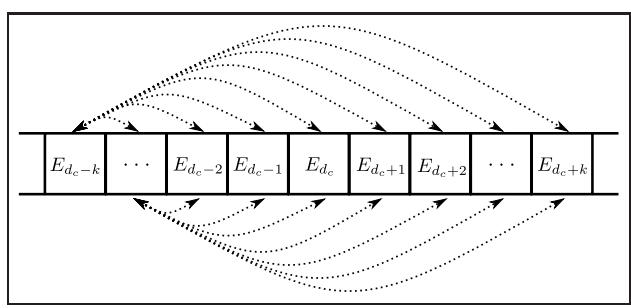

(e) $\mathrm{SM}(k=r(t))$

Fig. 2 Clustering methods

(8) If $i<d_{c}+r(t)$, go back to the step 2. Otherwise stop the procedure.

At each step in LCO, each clustering method is chosen at random from those methods, and chosen one is used in the step for generating the better neighbor.

\subsubsection{Clustering Radius}

In LCO, the solution is iteratively operated by a local clustering. Since the clustering radius decides the local solution size, its definition affects on the accuracy of the solution. That means, if the radius is set to smaller one, the accuracy of the solution sometimes becomes worse because the widely covered optimization in the whole solution is not performed. On the other hand, it is serious that the bigger radius causes the delay of computational time. Described in the section above, each clustering method generates a number of the neighborhood solution. In other words, each clustering method (especially SM) requires a longer computational time than operators used in SA to generate neighborhood solutions.

Thus, the definition of the clustering radius becomes important to generate a solution which has highly approximate accuracy with fast computational time.

\subsubsection{Some Remarks on LCO}

In the optimization procedure of each clustering method, replacing $S_{c}$ with $S_{n}$ is executed only when $C\left(S_{n}\right)$ is less than $C\left(S_{c}\right)$. This type of improvement is called "hill climbing improvement". On the other hand, SA suggests that it does not embrace the hill climbing improvement. Though LCO avoids convergence to the local optimal solution by using some different types of operations, it is not enough to converge to the widely spreaded local optimal solution by using the hill climbing improvement.

Also, there is a problem as well as SM referred above. Though SM is a much better effective operation for optimization, the computational time of SM is 10 times longer than others. In other words, using SM should be avoided by these two reasons.

\section{Hybrid Algorithm}

We propose a hybrid algorithm for JSP. The proposed method is developed by combining LCO with SA.

In the previous section, it is insisted SA is a good method for generating the high accurate solution and LCO is a good method for generating the good solution with fast computation time. Therefore, by combining SA with LCO, there are some possibilities to establish a better algorithm which can generate the high accurate solution with fast computation time.

In the following, we describe the new optimization procedure and discuss the expected effects of the proposed method.

\subsection{Optimization Procedure}

Some notations are added to use in proposed optimization procedure. 

$T(t) \quad$ : the temperature in $t^{\text {th }}$ step
$r(t)$ : the clustering radius in $t^{\text {th }}$ step
$S_{b} \quad$ : the best solution
$S_{c} \quad$ : the current solution
$S_{n} \quad$ : the neighborhood solution
$C(S) \quad$ : the cost of a solution $S$

The proposed algorithm is described as follows;

(1) Set $t=0$.

(2) Initialize $S_{c}$ at random.

(3) Set $S_{b}=S_{c}$.

(4) Select a set of consecutive elements in $S_{c}$ whose size is equal to $2 r(t)+1$.

(5) To create $S_{n}$, apply a clustering operation to the selected set.

(6) Set $\Delta_{n c}=C\left(S_{n}\right)-C\left(S_{c}\right)$ and $\Delta_{n b}=C\left(S_{n}\right)-$ $C\left(S_{b}\right)$.

(7) If $\Delta_{n b}<0$, replace $S_{b}$ and $S_{c}$ with $S_{n}$.

(8) If $\Delta_{n c} \leq 0$, replace $S_{c}$ with $S_{n}$. Otherwise generate an uniform random value $P(\forall P \in \mathbb{R}, 0 \leq$ $P \leq 1)$ and replace $S_{c}$ with $S_{n}$ if $P^{\prime}<P$. Here, $P^{\prime}$ is defined as;

$$
P^{\prime}=\exp \left\{-\frac{\Delta_{n c}}{T(t)}\right\}
$$

(9) Replace $t$ with $t+1$.

(10) If terminate conditions are satisfied, stop this procedure with the solution $S_{b}$. Otherwise go back to step 4 .

In the procedure, at each step, we use a clustering operation selected stochastically among five operations, SEM, SYM, IEM, SSM and SM, as same as LCO.

\subsection{Expected Effects}

The objective of the proposed method is to implement the SA mechanism, which accepts worse $S_{n}$ than $S_{c}$, with LCO. Fig. 3 indicates the optimizing results of the simple hill climbing algorithm $(\mathrm{HC})$ and SA for the $10 \times 10$ problem. $\mathrm{HC}$ is almost the same method as SA except $S_{c}$ is not replaced with $S_{n}$ when $S_{n}$ is worse than $S_{c}$. Generally, such an improvement mechanism of $\mathrm{HC}$ is so-called "hill climbing improvement". According to Fig. 3, the solution generated by $\mathrm{HC}$ is obviously worse than one generated by $\mathrm{SA}$. In fact, the hill climbing improvement has a risk that the solution generated by it easily falls into a local optimum when the problem has more than one peaks (local optima). The hill climbing improvement is known as a highly effective mechanism in terms of the convergence. On the other hand, it is also fundamentally true that $\mathrm{HC}$ makes the solution worse because it does not have a mechanism to avoid the solution falling into the local optima.

Though LCO is based on the hill climbing improvement, it is known that LCO is an effective method. In SA, the optimization is operated by some neighborhood operations based on the random swapping. And it sometimes causes some wasted searches, in which the same solution appears repeatedly. On

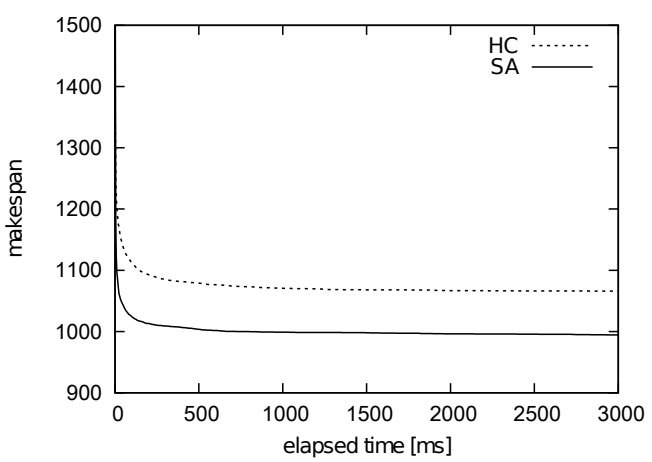

Fig. $3 \mathrm{HC}$ and SA

the other hand, because the same solution is seldom searched in a clustering process, there are few wasted searches in LCO. Also, the transition from $S_{c}$ to $S_{n}$ is frequently repeated in the early search of the optimization. Therefore, LCO is more efficient than SA in the early part of the optimization, and this property is a high advantage especially to solve large scale problems.

In summarizing those, the proposed method is expected to have the following properties.

- It derives a more highly accuracy approximate solution than LCO.

- It is more efficient than SA in the early part of the optimization.

\section{Numerical Experiments}

In this section, we evaluate the effectiveness of the proposed method by comparing it with LCO and SA. The numerical experiments on 4 pattern problems which are classified by their size are performed.

\subsection{Instance}

Table 2 shows instances used in this study. There are four items showing the problem name, the number of jobs, the number of machines and the optimal makespans. In this study, we use the widely known JSP benchmarks, some $10 \times 10$ problems and some others known as "10 tough problems". Because they are too hard to obtain the optimal solution, some do not derive their optimal makespans. In Table 2, the optimum is marked with the asterisk. That means some problems have not been proved to find its optimal makespan. The value shown as the optimum is the upper-bound of the optimal makespan. In addition, in Table 2, the randomly generated problem by us is included. Since the random problem has 20 jobs and 50 machines, its size is equal to 1000 and we regard it as a large scale problem. Also it has no proved optimal solution.

\subsection{Experimental Conditions}

Table 3 indicates the detail of experimental conditions. In Table $3, l$ means the problem size and is defined as eq. (12);

$$
l=n \times m
$$


Table 2 The instance used in this study

\begin{tabular}{|c|c|c|c|}
\hline Name & Jobs & Machines & Optimal makespan \\
\hline ABZ6 & 10 & 10 & 943 \\
\hline ORB4 & 10 & 10 & 1005 \\
\hline ORB5 & 10 & 10 & 887 \\
\hline LA27 & 20 & 10 & 1235 \\
\hline LA29 & 20 & 10 & 1152 \\
\hline ABZ7 & 20 & 15 & 656 \\
\hline ABZ8 & 20 & 15 & $665^{*}$ \\
\hline random & 20 & 50 & - \\
\hline
\end{tabular}

Table 3 Experimental conditions

\begin{tabular}{|c|c|c|c|c|}
\hline \multicolumn{2}{|c|}{} & LCO & SA & Proposed \\
\hline \multirow{4}{*}{ termination time $[\mathrm{s}]$} & (a) & 10.0 & 10.0 & 10.0 \\
\cline { 2 - 5 } & (b) & 30.0 & 30.0 & 30.0 \\
\cline { 2 - 5 } & (c) & 50.0 & 50.0 & 50.0 \\
\cline { 2 - 5 } & (d) & 100.0 & 100.0 & 100.0 \\
\hline SEM:SIM:SYM:IEM:SM & $3: 3: 2: 1: 1$ & - & $3: 3: 2: 1: 1$ \\
\hline \multirow{3}{*}{ clustering radius } & $\frac{1}{3} l, \frac{2}{3} l$ & - & $\frac{1}{3} l, \frac{2}{3} l$ \\
\hline \multirow{3}{*}{ initial temperature } & (a) & - & 1.0 & 1.0 \\
\cline { 2 - 5 } & (b) & - & 10.0 & 10.0 \\
\cline { 2 - 5 } & (c) & - & 100.0 & 100.0 \\
\cline { 2 - 5 } & (d) & - & 10.0 & 10.0 \\
\hline \multicolumn{2}{|c|}{ change rate of temp. } & - & 0.99 & 0.99 \\
\hline \multicolumn{2}{|c|}{ initial iteration } & - & 500 & - \\
\hline \multicolumn{2}{c}{ change rate of iteration } & - & 1.05 & - \\
\hline \multicolumn{2}{|c|}{ iteration limit } & - & 1000 & - \\
\hline
\end{tabular}

$n:$ the number of jobs

$m$ : thenumber of machines

We define four patterns for the termination time and the initial temperature. These patterns are determined according to the problem size to make experiments easier.

The program is coded by Java and not coded for parallel computing. Also, it is run on Intel $®$ Pentium $®$ $4(2.80 \mathrm{GHz})$ for the benchmarks and Intel $® \mathrm{CORE}^{\mathrm{TM}}$ i5 $(3.30 \mathrm{GHz})$ for the random problem.

The experiment is performed 100 times for each problem. And each method is compared using the basic statistical data about the solution derived by them.

\subsection{Computational Results}

Table 4 indicates results of numerical experiments. In Table 4, the accuracy of makespan is defined as eq. (13);

$$
\begin{aligned}
\text { accuracy } & =\frac{M_{g}}{M_{o}} \\
M_{g} & : \text { makespan of generated solution } \\
M_{o} & : \text { optimal makespan }
\end{aligned}
$$

According to Table 4, the proposed method can search for better solutions than LCO at the accuracy. This result is natural because the proposed method becomes more difficult to fall into the local optima than LCO by combining it with SA. In this point,

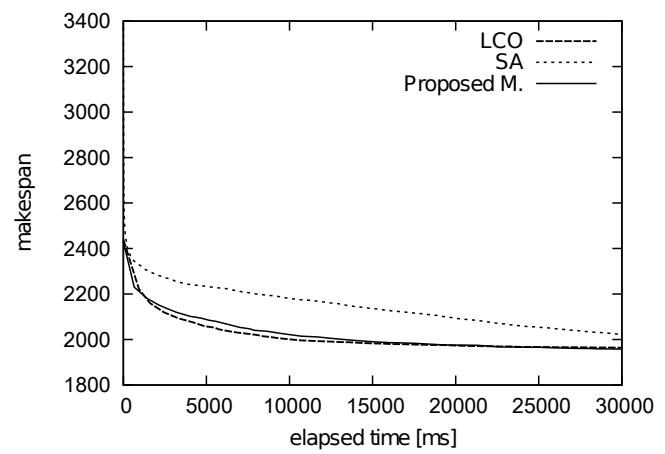

Fig. 4 The convergence of the solution

as we expected, the proposed method is an improved algorithm of LCO. On the other hand, when the problem size becomes larger and the computational time becomes longer, the proposed method derives worse solution than SA. To examine the result in detail, we analyze the convergence of the solution.

Fig. 4 shows the convergence of the solution in the early part of the optimization for the random problem. From Fig. 4, LCO and the proposed method can search good solutions faster than SA. It means they are more efficient than SA in the early search of the optimization. In particular, in the early search of the optimization, the proposed method can obtain good solutions faster than SA if the conditions about the temperature are almost same. This result shows that LCO and the proposed method is profitable in the case the computational time is limited. It is the important property for the large scale problems because, in general, planning time for making schedules is limited in the real industrial world.

In this section, we describe an effect of the proposed method by comparing performance with fundamental algorithms. From the results of the experiments, the proposed method is proved under below.

- It can search better solutions than LCO.

- It can search good solutions faster than SA in the early part of the optimization.

Therefore, the proposed method has both performance between LCO and SA. And it is especially effective from the early search of the optimization to the middle one. To solve large scale problems, this property is very important because it can largely reduce computational time.

\section{Conclusion}

This study is summarized as follows;

- This study proposes a hybrid solution for JSP.

- The proposed method is based on SA and LCO.

- As for the accuracy of the solution, the proposed method is more effective than LCO.

- In the early search of the optimization, the proposed method can search good solutions faster than SA.

- The proposed method is effective from the early part of the optimization to the middle one. And 
Table 4 The computational results

\begin{tabular}{|c|c|c|c|c|c|c|}
\hline \multirow{2}{*}{ Name } & \multicolumn{2}{|c|}{ Used } & \multicolumn{4}{|c|}{ makespan } \\
\hline & method & condition & Avg. (accuracy) & Var. & Min. & Max. \\
\hline \multirow{3}{*}{ ABZ6 } & $\mathrm{SA}$ & (a) & $957.7(1.016)$ & 100.99 & 943 & 979 \\
\hline & $\mathrm{LCO}$ & (a) & $1002.22(1.063)$ & 857.35 & 943 & 1073 \\
\hline & Proposed & (a) & $\mathbf{9 5 7 . 1}(1.015)$ & 45.25 & 946 & 976 \\
\hline \multirow{3}{*}{ ORB4 } & $\mathrm{SA}$ & (a) & $1044.39(1.039)$ & 388.38 & 1006 & 1109 \\
\hline & $\mathrm{LCO}$ & (a) & $1095.76(1.090$ & 978.32 & 1042 & 1179 \\
\hline & Proposed & (a) & $\mathbf{1 0 4 0 . 1 2 ( 1 . 0 3 5 )}$ & 45.01 & 1019 & 1053 \\
\hline \multirow{3}{*}{ ORB5 } & $\mathrm{SA}$ & (a) & $\mathbf{9 3 6 . 2 4}(1.056)$ & 612.72 & 890 & 1015 \\
\hline & $\mathrm{LCO}$ & (a) & $1023.86(1.154)$ & 1741.98 & 926 & 1126 \\
\hline & Proposed & (a) & $938.97(1.059)$ & 191.85 & 909 & 973 \\
\hline \multirow{3}{*}{ LA27 } & $\mathrm{SA}$ & (b) & $\mathbf{1 2 7 4 . 5 2}(1.032)$ & 131.030 & 1254 & 1308 \\
\hline & $\overline{\mathrm{LCO}}$ & (b) & $1400.41(1.134)$ & 1690.94 & 1303 & 1490 \\
\hline & Proposed & (b) & $1323.28(1.071)$ & 99.26 & 1300 & 1348 \\
\hline \multirow{3}{*}{ LA29 } & $\mathrm{SA}$ & (b) & $\mathbf{1 2 5 1 . 6 0}(1.086)$ & 524.98 & 1211 & 1307 \\
\hline & $\mathrm{LCO}$ & (b) & $1357.64(1.179)$ & 1526.83 & 1267 & 1479 \\
\hline & Proposed & (b) & $1281.18(1.112)$ & 115.61 & 1250 & 1311 \\
\hline \multirow{3}{*}{ ABZ7 } & $\mathrm{SA}$ & (c) & 695.84( 1.061$)$ & 94.19 & 676 & 723 \\
\hline & $\mathrm{LCO}$ & (c) & $760.03(1.159)$ & 253.71 & 727 & 807 \\
\hline & Proposed & (c) & $730.57(1.114)$ & 32.93 & 707 & 742 \\
\hline \multirow{3}{*}{ ABZ8 } & $\mathrm{SA}$ & (c) & 699.97 ( 1.053$)$ & 63.85 & 681 & 730 \\
\hline & LCO & (c) & $775.28(1.166)$ & 260.96 & 739 & 816 \\
\hline & Proposed & (c) & $742.05(1.116)$ & 27.77 & 727 & 752 \\
\hline \multirow{3}{*}{ random } & $\mathrm{SA}$ & (d) & 1852.27 & 414.20 & 1814 & 1894 \\
\hline & $\mathrm{LCO}$ & (d) & 1957.22 & 821.53 & 1897 & 2020 \\
\hline & Proposed & (d) & 1929.79 & 251.63 & 1866 & 1970 \\
\hline
\end{tabular}

this property is available for a large scale scheduling problem.

- The proposed method is able to search a good schedule efficiently without falling into the local optimal solution.

- To guarantee more effective search on the proposed method, we should adjust parameters depending on each problem and the biggest problem is that adjusting the parameters is very troublesome.

\section{References}

[1] M. R. Garey and D. S. Johnson: Computers and Intractability - A Guide to the Theory of NPCompleteness, Freeman and Company (1979)

[2] R. Nakano and T. Yamada: Conventional genetic algorithm for job shop problems; Proceedings of The Fourth International Conference on Genetic Algorithms, pp. 474-479 (1991)

[3] P. J. M. van Laarhoven, E. H. L. Aarts and J. K. Lenstra: Job shop scheduling by simulated annealing; Operations Research, INFORMS, p. 113-125 (1992)

[4] M. Furukawa, M. Watanabe and Y. Matsumura: Lcoal Clustering Organization (LCO) solving a largescale TSP; Journal of Robotics and Mechatronics, Vol. 17, No. 5, p. 560 (2005)

[5] M. Furukawa, Y. Matsumura and M. Watanabe: Development of local clustering organization applied to job-shop scheduling problem; Journal of the Japan
Society for Precision Engineering (CD-ROM), Vol 72, No. 7, p. 867-872 (2006)

[6] Y. Konno and K. Suzuki: Performance of extended Local Clustering Organization (LCO) for large scale Job-shop Scheduling Problem (JSP); IEEJ Transactions on Electronics, Information and Systems, Volume 129, Issue 7, pp. 1363-1370 (2009)

[7] T. Kohonen: The self-organizaing map; Neurocomputing, Vol. 21, No. 1-3, pp. 1-6 (1998)

\section{著 者 略歴}

\section{声符㸃康将}

1989 年 6 月 12 日生. 2012 年北海道大学工学部情報工 レクトロニクス学科卒業. 同年 4 月より北海道大学大学院情 報科学研究科修士課程に進学し, 現在に至る.組合せ最適化 問題の研究, とくにジョブショップスケジューリング問題に 対するメタヒューリスティクスの適用などの研究に従事.工 学学士. 日本機械学会の学生員, 精密工学会の Web 級学生 会員.
鈴呆育势
論文誌 Vol. 26, No. 3, p. 109 参照
山本势雅
論文誌 Vol. 26, No. 3, p. 109 参照
就乫郝企

論文誌 Vol. 26, No. 3, p. 109 参照 